Pecvnia, 8 (2009), pp. 235-262

\title{
Características del conocimiento transferido como determinantes del rendimiento de los sistemas de franquicia
}

Recibido: Enero 2009

Aceptado: Septiembre 2009

\author{
Beatriz Minguela Rata \\ minguela@ccee.ucm.es \\ $M^{a}$ Concepción Rodríguez Benavides \\ mc.rb@ccee.ucm.es \\ José Ignacio López Sánchez \\ jilopez@ccee.ucm.es \\ Universidad Complutense de Madrid \\ Departamento de Organización de Empresas \\ Fac. de Ciencias Económicas y Empresariales \\ Campus de Somosaguas \\ 28223 Pozuelo de Alarcón - Madrid (España)
}

En este trabajo se pretende analizar la influencia de la naturaleza tácita y el valor del conocimiento (dos características del conocimiento determinantes de la facilidad con la que se puede transferir el mismo) en el rendimiento de los sistemas de franquicia. Se ha llevado a cabo un análisis de regresión lineal sobre una muestra de establecimientos franquiciados en diferentes cadenas de franquicia y sectores de actividad que operan en España, midiendo el rendimiento tanto de manera objetiva como subjetiva y tomando el tipo
In this paper the influence of tacitness and value of knowledge on the performance of franchise systems are studied. With this aim, a linear regression analysis is conducted on a sample of franchisee of different franchise chains and sectors of activity operating in Spain. In the model we added a control variable, named transformation, which represents the kind of activities carried out in the franchisee units: just commercial or transformative and commercial activities. The findings show that 
de actividad llevada a cabo por el establecimiento franquiciado como variable de control. Los resultados parecen indicar que la naturaleza tácita del conocimiento influye de manera negativa en el rendimiento de los sistemas de franquicia mientras que el valor del conocimiento afecta de manera positiva.

Palabras clave: Naturaleza tácita, valor del conocimiento, rendimiento, sistemas de franquicia, franquiciado. tacit knowledge has a negative impact on franchise systems performance, the value of knowledge affects it in a positive manner whereas the control variable is significant.

Key words: Tacit knowledge, value of knowledge, performance, franchise systems, franchisee.

\section{INTRODUCCIÓN}

La forma en la que las organizaciones perciben el conocimiento ha cambiado en los últimos tiempos. La consideración de que el conocimiento sólo reside en los individuos, que los expertos son la principal fuente de conocimiento y que se trata de un activo que puede ser almacenado y recuperado cuando sea necesario, ha sido relegada a un segundo plano. Ahora, son numerosas las organizaciones que perciben el conocimiento de forma más amplia y completa al reconocer que se trata de un fenómeno social, distribuido y dinámico.

Esta consideración del conocimiento convierte a la transferencia del mismo en un proceso fundamental para el éxito de las organizaciones. En ellas, siempre se han llevado a cabo transferencias de conocimiento ya que, constantemente, los trabajadores están intercambiando conocimiento. Sin embargo, las transferencias que forman parte del día a día de la organización son de tipo espontáneo, informal y local y, dada la importancia que el conocimiento tiene para las organizaciones, éstas se ven obligadas a diseñar procesos de transferencia más estructurados y deliberados (O'Dell y Grayson 1998; Davenport y Prusak 2001; Dixon 2001; Husted y Michailova 2002).

De hecho, para muchas organizaciones, una de las principales preocupaciones es la de intentar maximizar la utilidad del conocimiento lo cual las lleva a reconocer que el proceso de transferencia es una importante fuente de ventajas competitivas (Contractor y Kundu 1998; Holtshouse 1998; Osterloh y Frey 1999; Teece 2000; Cowan, Soete y Tchervonnaya 2001; Dixon 2001; Schulz y Jobe 2001; Subramaniam y Venkatraman 2001; Von Krogh, Nonaka y Aben 2001; Husted y Michailova 2002). 
En concreto, la transferencia de conocimiento puede influir positivamente en el rendimiento de las organizaciones porque permite sustituir las prácticas ineficientes por otras que han demostrado una mayor eficiencia (Szulanski 1996), replicar las mejores prácticas por toda la organización (Lubit 2001; Schulz y Jobe 2001) e identificar y explotar economías de escala y de alcance relativas al conocimiento (Schulz y Jobe 2001; Husted y Michailova 2002). También, facilita la integración y la coordinación entre los miembros de la organización dado que incrementa el solapamiento del conocimiento de los individuos (Grant 1996a, 1996b; Nonaka, Reinmoeller y Senoo 1998; Nonaka, Toyama y Konno 2000; Lubit 2001; Schulz y Jobe 2001), y estimula la innovación al favorecer el aprendizaje mutuo y la cooperación (Tsai 2001).

Asimismo, la transferencia de conocimiento puede dificultar la imitación del mismo al incrementar la base de conocimiento (Schulz y Jobe 2001). Y, además, las transferencias externas de conocimiento permiten acceder a conocimiento que puede ser necesario para la empresa (Dyer y Nobeoka 2000; Subramaniam y Venkatraman 2001; Foss y Pedersen 2002; Kotabe, Martin y Domoto 2003).

En este trabajo pretendemos estudiar la transferencia que se produce en los sistemas de franquicia, un acuerdo de colaboración que en los últimos tiempos ha experimentado un importante crecimiento en España y para el que dicho proceso es fundamental.

La explotación de los establecimientos franquiciados precisa de la transferencia de conocimiento desde el franquiciador a estos últimos porque, al caracterizarse la franquicia por la reproducción exacta de un concepto de negocio, en todos los establecimientos se debe hacer uso del mismo conocimiento.

Por tanto, dada la importancia que tiene para los sistemas de franquicia el proceso de transferencia de conocimiento en el sentido en que cualquier dificultad que se produzca en el proceso de transferencia tendrá repercusiones negativas en el rendimiento de la cadena de franquicia, el propósito de esta investigación es el de analizar la influencia de dos características del conocimiento determinantes de la facilidad con la que se puede transferir el mismo (la naturaleza tácita y el valor), en el rendimiento de los sistemas de franquicia. 


\section{FUNDAMENTO TEÓRICO}

\subsection{El rendimiento de los sistemas de franquicia}

El rendimiento de los sistemas de franquicia presenta múltiples facetas y niveles (Elango y Fried 1997) en el sentido en que este rendimiento puede relacionarse con numerosas dimensiones y que por tanto, sea necesario considerar, no una, sino varias medidas del mismo. Así, por ejemplo, para medir el crecimiento del sistema de franquicia se pueden utilizar como indicadores el número de establecimientos y la rentabilidad de los mismos. Sin embargo, es posible que el franquiciador consiga crecer rápidamente en tamaño, vía cantidad de establecimientos, pero ser incapaz de mantener la rentabilidad de los mismos.

En cuanto a los múltiples niveles del rendimiento de los sistemas de franquicia, éstos representan la perspectiva del análisis. De esta forma, el rendimiento puede ser analizado desde el punto de vista del franquiciador, del franquiciado, de los trabajadores, de los clientes, de la sociedad en su conjunto... y al igual que sucedía con las múltiples facetas, las medidas utilizadas para los diferentes niveles, también pueden ser contradictorias. Un ejemplo es el de las implicaciones que tiene para el franquiciador y para un franquiciado la apertura de nuevas unidades operativas. El incremento en el número de establecimientos del sistema beneficia al franquiciador porque recibe más ingresos vía cánones de entrada y royalties, pero puede perjudicar al franquiciado si la incorporación de nuevas unidades supone una amenaza para su mercado.

Por tanto, en la literatura no hay un consenso sobre la forma más idónea de medir el rendimiento de los sistemas de franquicia. Pueden emplearse diversas fuentes de información, uno o varios indicadores cuyos criterios sean, o no, económico-financieros y además, la medición puede realizarse de manera objetiva o subjetiva.

Según Anderson (1984), Sorenson y Sorensen (2001) así como Bradach (1998), el beneficio de cada establecimiento es el indicador del rendimiento más apropiado. Sin embargo, los problemas que plantea el acceso a los datos relativos a los beneficios obliga a buscar medidas alternativas relacionadas con los ingresos y los costes.

De este modo, Anderson (1984), Martin (1988), Norton (1988a,b) así como Sorenson y Sorensen (2001) utilizan las ventas como medida del rendimiento de los sistemas de franquicia. En realidad, tanto las ventas como el número de unidades del sistema son dos medidas del 
rendimiento relacionadas con los ingresos. $Y$ es que, las ventas de un establecimiento son difíciles de incrementar a partir de un determinado nivel ya que la proximidad a los clientes con la intención de buscar su comodidad es característico de la localización y, una vez que la localización ha sido elegida, es muy difícil cambiar la comodidad para estimular unas mayores ventas. De ahí que las limitaciones económicas inherentes a toda localización expliquen por qué los nuevos establecimientos alcanzan rápidamente su máximo volumen.

La dificultad que plantea el incremento de las ventas en los establecimientos ya en funcionamiento, obliga a incorporar nuevas unidades al sistema. En este sentido, Castrogiovanni, Justis y Julian (1993) así como Martin y Justis (1993) emplean el número total de establecimientos. Esta medida permite, en primer lugar, incrementar la presencia de la marca en el mercado, lo cual beneficia a la cadena al ser una forma de publicidad que refuerza su identidad. $Y$, en segundo lugar, el franquiciador puede reducir los costes en los que ha incurrido en los primeros años al tratar de generar el conocimiento que le permitiera replicar su negocio porque puede repartirlos entre un mayor número de unidades ${ }^{1}$.

Para Szulanski, Jensen y Lee (2001), el crecimiento es la medida fundamental de éxito de los sistemas de franquicia, pero sólo es válido en las primeras etapas del sistema porque es cuando el franquiciador genera mayores ingresos por cánones de entrada, royalties y venta de equipos a los franquiciados como consecuencia de la apertura de nuevos establecimientos. En cambio, posteriormente, cuando el mercado empieza a saturarse o el sistema ha alcanzado la madurez, el franquiciador tiende a maximizar las unidades que ya posee y, en este caso, el crecimiento del sistema como medida del rendimiento puede presentar problemas.

Thompson (1994) así como Darr, Argote y Epple (1995) consideran la productividad como medida del rendimiento de los sistemas de franquicia, mientras que Fenwick y Strombom (1998) lo miden a partir del volumen de negocio, la rentabilidad y el crecimiento. Por su parte, Bates (2002), como medida del rendimiento, utiliza la rentabilidad del franquiciador y para ello, determina los siguientes indicadores: ingresos netos, ingresos netos por trabajador, ingresos netos por localidad, beneficios (antes de impuestos) como un porcentaje de los ingresos y los pagos realizados por los franquiciados como un porcentaje de los ingresos. 
Finalmente, Carman y Klein (1986) reconocen que en el estudio del rendimiento de los sistemas de franquicia, se debería tener en cuenta los intereses de los consumidores, el franquiciador, los franquiciados y otros segmentos de la sociedad que están afectados por la franquicia. No obstante, dichos intereses pueden ser agrupados en siete grandes áreas: 1) sensibilidad al cambio, 2) innovación, 3) eficiencia funcional, 4) eficiencia transaccional, 5) rentas como un incentivo al rendimiento, 6) equidad y 7) externalidades.

En definitiva, el rendimiento de los sistemas de franquicia es una variable que presenta importantes problemas en cuanto a su medición. No obstante, la revisión de la literatura nos permite identificar múltiples formas de medir el rendimiento, aunque la mayoría de los indicadores utilizados en los trabajos empíricos están relacionados con las ventas y el número de establecimientos.

\subsection{La naturaleza tácita del conocimiento}

La dimensión tácita del conocimiento es aquella parte del mismo que los individuos pueden explicar a través de un conjunto de reglas articuladas, mientras que, la dimensión implícita, inarticulada o explícita, comprende aquella parte del conocimiento que los individuos no pueden expresar porque está basado en unas reglas que no conocen de forma consciente (Polanyi 1962, 1966).

La parte explícita del conocimiento es formal y sistemática por lo que puede ser comunicada a través de símbolos desde su poseedor a otras personas y, el receptor de dicha comunicación, puede llegar a tener tanto conocimiento como el emisor. En cambio, el componente tácito es intuitivo, inarticulado, se adquiere a través de la experiencia y no puede ser explicado por los individuos, de ahí que sea más difícil de comunicar y compartir con otros (Winter 1987; Nonaka 1991; Garud y Nayyar 1994; Hedlund 1994; Von Hippel 1994; Nonaka y Takeuchi 1995; Grant 1996a, 1997; Nonaka, Toyama y Konno 2000; Ambrosini y Bowman 2001; Davenport y Prusak 2001; Lubit 2001; Nielsen 2001; Schulz 2001; Schulz y Jobe 2001; Hall y Andriani 2002; Chen 2004).

El hecho de que una parte del conocimiento sea tácita y otra explícita y de que el conocimiento tácito presente mayores problemas de formalización, expresión y comunicación que el conocimiento explícito, afecta al proceso de transferencia de dicho conocimiento. 
En este sentido, Galunic y Rodan (1998) reconocen que la transferencia de conocimiento tácito es difícil porque suele requerir la movilización de los individuos que poseen ese conocimiento. $\mathrm{Y}$, para Empson (2001), el conocimiento tácito es difícil de transferir porque, en primer lugar, no puede ser articulado de forma escrita o verbal, aunque sí puede ser aprendido mediante la experiencia y, en segundo lugar, porque es específico a un contexto y reside en los sistemas organizativos.

Igualmente, tanto Nonaka y Takeuchi (1995) como Nonaka, Toyama y Konno (2000) admiten que el carácter personal e intuitivo del conocimiento tácito es lo que lo hace difícil de transferir, mientras que, por el contrario, el conocimiento explícito puede ser transferido con relativa facilidad. También, Davenport y Prusak (2001) consideran que el conocimiento que es más o menos explícito puede ser incorporado en documentos y bases de datos, lo cual permite transferirlo con una precisión razonable. En cambio, la transferencia de conocimiento tácito es difícil y requiere un alto contacto personal. De ahí que, las organizaciones interesadas en la transferencia de conocimiento tácito establezcan programas de formación y utilicen la tecnología de la información para permitir que los individuos compartan el conocimiento.

Para Grant (1996b, 1997), la transferencia de conocimiento tácito es más difícil que la del conocimiento explícito porque el conocimiento tácito sólo puede ser observado a través de su aplicación, mientras que, el conocimiento explícito se caracteriza por la facilidad de comunicación. Y, según Maleaba y Orsenigo (2000), Teece (2000), así como Lubit (2001), la dificultad de expresar y codificar el conocimiento tácito lo hace más difícil de transferir que el conocimiento explícito.

Winter (1987) también reconoce que el conocimiento tácito es difícil de transferir, aunque, dicha dificultad, es menor cuando el conocimiento tácito puede ser enseñado ya que, el que un individuo desempeñe una tarea según las pautas indicadas por otro individuo, permite reducir el tiempo y facilita el aprendizaje de la tarea. De este modo, a diferencia del conocimiento tácito, el conocimiento articulable es más fácil de transferir, pero, esa facilidad se puede reducir cuando el conocimiento articulable no está articulado o codificado, como es el caso del diseño de un producto que con el tiempo ha sufrido modificaciones y el documento en el que se registró simbólicamente ese diseño no se ha actualizado. 
En consecuencia, el grado de dificultad del proceso de transferencia de conocimiento se ve afectado por el hecho de que el conocimiento tácito se pueda o no enseñar y porque el conocimiento articulable esté o no articulado.

En definitiva, mientras que una parte del conocimiento es explícita, otra parte permanece tácita y, cuanto mayor sea esta última, más difícil será la transferencia de conocimiento. En consecuencia, como la naturaleza tácita está positivamente relacionada con la dificultad en la transferencia de conocimiento, y ésta última afecta de manera negativa al rendimiento de los sistemas de franquicia, podemos formular la primera hipótesis de esta investigación en los siguientes términos:

Hipótesis 1

La naturaleza tácita del conocimiento influye negativamente en el rendimiento de los sistemas de franquicia.

\subsection{El valor del conocimiento}

El concepto de valor hace referencia a la utilidad de las cosas para satisfacer las necesidades o proporcionar bienestar y también, a la cualidad de las cosas, en virtud de la cual se está dispuesto a pagar un dinero o equivalente por poseerlas.

En el caso concreto del conocimiento, Gupta y Govindarajan (2000) sólo consideran la primera acepción, esto es, el conocimiento valioso es aquél que tiene una utilidad para quien lo recibe. Por tanto, del mismo modo que estos autores, a efectos de este trabajo consideramos que el valor del conocimiento se refiere a la utilidad que tiene para quien lo recibe, que, en nuestro caso, es el franquiciado.

Respecto a la transferencia de conocimiento, estos mismos autores reconocen que el conocimiento valioso es más fácil de transferir porque para los posibles receptores resulta más atractivo. Por tanto, como el valor está negativamente relacionado con la dificultad en la transferencia de conocimiento, y ésta última afecta de manera negativa al rendimiento de los sistemas de franquicia, formulamos la siguiente hipótesis: 
Hipótesis 2

El valor del conocimiento influye positivamente en el rendimiento de los sistemas de franquicia.

Con independencia de las dos hipótesis planteadas, puede ser interesante analizar la influencia conjunta de ambas variables sobre el rendimiento de los sistemas de franquicia. En este sentido formulamos la siguiente hipótesis:

Hipótesis 3

Cuanto menos tácito y más valor del conocimiento, mayor rendimiento de los sistemas de franquicia.

\section{METODOLOGÍA}

\subsection{Descripción de la población}

Para analizar la relación anterior, se diseñó un cuestionario (a partir de la revisión de la literatura tanto teórica como empírica) agrupando preguntas referentes a cada una de las variables objeto de estudio. Dicho cuestionario fue enviado a la población objetivo que estaba constituida por 267 establecimientos franquiciados localizados en España, que desarrollan su actividad en diferentes sectores y que son miembros de la Asociación Española para el Desarrollo y Defensa del Franquiciado (AEDEF).

Se decidió adoptar la perspectiva de análisis del franquiciado porque la mayoría de los estudios en materia de franquicia solicitan la información a los franquiciadores $\mathrm{y}$, sobre todo, porque creemos que es más interesante y objetivo obtener la información de aquéllos que tienen que aplicar el conocimiento desarrollado por el franquiciador. A continuación pondremos de manifiesto cómo se han construido los indicadores representativos de las variables objeto de estudio. 


\subsection{Construcción de las variables}

\section{El rendimiento}

Para medir el rendimiento de los sistemas de franquicia, se ha tenido en cuenta la recomendación de Elango y Fried (1997) sobre el uso de múltiples indicadores. Así, en primer lugar, mediremos el rendimiento a partir de las ventas generadas en cada establecimiento franquiciado. Tal y como reconocen Flechoso Sierra (1997), Sorenson y Sorensen (2001) y Tormo (2002) las ventas son un buen indicador del rendimiento. No obstante, aunque en muchos estudios empíricos se obtienen las ventas por establecimiento, entendemos que, dado que los establecimientos suelen tener distinta capacidad, es más adecuado el uso de los metros cuadrados de cada establecimiento. Por tanto, uno de los indicadores que utilizaremos para medir el rendimiento de los sistemas de franquicia es la ratio de las ventas generadas, en 2005, por metro cuadrado (VTAS/m2).

Una segunda medida, ahora subjetiva, consiste en valorar, en una escala de Likert de 1 a 7 , la importancia (1: nada importante; 7: sumamente importante) que tienen una serie de indicadores así como el grado de satisfacción (1: totalmente insatisfecho; 7: totalmente satisfecho) obtenido durante el último año. De este modo, la escala de medida será la suma de los productos de la importancia y la satisfacción (RDTO), donde una puntuación alta indica un mayor rendimiento.

Concretamente, el franquiciado valorará un total de 6 ítem relativos a 1) las ventas generadas por su establecimiento en 2005, 2) el tiempo de recuperación de la inversión inicial, 3) la evolución experimentada por el concepto de negocio de la franquicia en el año 2005, 4) la productividad de su establecimiento, 5) la competitividad de las condiciones de compra y aprovisionamiento facilitadas por el franquiciador en 2005 y 6) la equidad en el reparto de las ganancias con la central de franquicia en 2005.

Los dos primeros ítems se corresponden con los indicadores económicos que van a ser valorados cualitativamente. Con la evolución experimentada por el concepto de negocio de la franquicia en el año 2005, se pretende medir la sensibilidad al cambio y la innovación. La productividad permite medir la eficiencia operativa, mientras que, la competitividad de las condiciones de compra y aprovisionamiento facilitadas por el franquiciador en 2005 es un indicador de la eficiencia transaccional. Finalmente, con la equidad en el reparto de las ganancias en 2005, se 
pretende que el franquiciado valore la justicia en cuanto a la distribución de las rentas.

Entendemos conveniente hacer uso de medidas subjetivas porque éstas valoran el rendimiento del sistema según la percepción de los agentes, que en nuestro caso, es el franquiciado, y porque es muy probable que no todos los encuestados estén dispuestos a proporcionarnos los datos sobre las ventas y los metros cuadrados del establecimiento.

Es cierto que las medidas objetivas, como son las ventas, tienen una mayor validez, pero la literatura ha demostrado que existe una gran correlación entre las medidas objetivas y subjetivas del rendimiento, lo que hace que ambas sean válidas a la hora de analizar el rendimiento (Hart y Banbury 1994).

\section{La naturaleza tácita del conocimiento}

El enorme interés que desde el punto de vista teórico ha suscitado la naturaleza tácita del conocimiento, contrasta con el escaso tratamiento empírico que ha recibido.

Bresman, Birkinshaw y Nobel (1999) utilizan sólo la posibilidad de ser enseñado como variable de medida de la naturaleza tácita del conocimiento, mientras que Simonin (1999), Hansen (1999) y Schulz (2001), sólo tienen en cuenta el grado de codificación del conocimiento.

Sin embargo, Zander y Kogut (1995), basándose en el reconocimiento que hace Winter (1987) de que, aunque parte del conocimiento no pueda ser codificado, sí puede ser enseñado, determinan la medición de la naturaleza tácita del conocimiento a partir de dos variables: la posibilidad de estar codificado y la posibilidad de ser enseñado.

En nuestro trabajo, la medición de la naturaleza tácita del conocimiento (NTAC) se va a llevar a cabo a través de las dos dimensiones porque entendemos que se trata de una medida más completa. La primera es el grado de codificación que presenta el conocimiento transmitido por el franquiciador para que el franquiciado pueda explotar su establecimiento. La segunda es la posibilidad de ser enseñado, la cual hace referencia a la facilidad o dificultad con la que el conocimiento necesario para gestionar un establecimiento de franquicia puede ser enseñado. 
Respecto al grado de codificación, se preguntó al franquiciado su grado de acuerdo sobre si el conocimiento que le transmite el franquiciador está lo suficientemente descrito en los manuales de explotación. Por su parte, para la posibilidad de ser enseñado, se diseñaron cuatro ítems con los cuales se pretende valorar si el conocimiento puede ser aprendido a través del estudio de los manuales de explotación y a través de conversaciones con personal experimentado, así como si la formación inicial y la continua que el franquiciador le proporciona, es sencilla y rápida. Todos estos ítems fueron diseñados con una escala de Likert de 7 puntos (1: totalmente en desacuerdo, 7: totalmente de acuerdo). La variable naturaleza tácita se construyó a partir de la suma de todos los ítems mencionados. Un mayor valor de esta variable indica que el conocimiento es más explícito.

\section{El valor del conocimiento}

Para medir el valor del conocimiento (VALOR) tendremos en cuenta la efectividad y el carácter sustancial del mismo. Y es que, de acuerdo con Martínez (1988), el saber hacer debe ser comercialmente efectivo y permitir explotar correctamente un establecimiento y para Flechoso Sierra (1997), Pablos (1998) y Alonso Prieto (2003), el saber hacer del franquiciador es sustancial en el sentido en que debe facilitar a los nuevos franquiciados abrir su establecimiento de forma competitiva y a los franquiciados existentes, mejorar su rendimiento.

En consecuencia, mediremos el valor del conocimiento a través de dos ítems, con escala de Likert de 7 puntos (1: totalmente en desacuerdo; 7: totalmente de acuerdo) con los que se pretende valorar si el conocimiento que el franquiciador les transmite, facilita la apertura de su establecimiento y si posteriormente le permite mejorar su rendimiento. La medida empleada está formada por la suma de ambos ítems de tal forma que a mayor puntuación, más valioso es el conocimiento.

\section{Variable de control}

Finalmente, como la población objetivo estaba constituida por establecimientos que desempeñan su actividad tanto en el sector servicios como en el comercio minorista y en la hostelería y restauración, consideramos conveniente incluir una variable dummy (TRANSF), con el objetivo de observar si los establecimientos que desempeñan actividades 
de comercialización y transformación física presentan unos rendimientos diferentes a los de aquellos establecimientos donde solamente se llevan a cabo actividades comerciales. Para ello, se pidió a los encuestados que indicasen el sector de actividad de su marca o enseña así como si desempeñan alguna actividad de transformación física. A partir de esta información, la variable de control toma valor 1 si en el establecimiento se realiza algún tipo de transformación física, mientras que su valor será 0 si sólo se desempeñan actividades comerciales.

En el Cuadro 1, de manera recopilatoria, aparece la descripción de las variables consideradas en el estudio así como su tipología.

Cuadro 1: Variables utilizadas en el estudio

\begin{tabular}{|c|c|c|c|}
\hline VARIABLE & ACRÓNIMO & TIPO & TIPO \\
\hline Naturaleza tácita & NTAC & Independiente & Continua (acotada entre 1 y 7 ) \\
\hline Valor & VALOR & Independiente & Continua (acotada entre 1 y 7 ) \\
\hline Transformación & TRANSF & Control & Dicotómica \\
\hline Ventas por metro cuadrado & $\mathrm{VTAS} / \mathrm{m} 2$ & Dependiente & Continua (euros/m2) \\
\hline $\begin{array}{l}\text { Rendimiento (medido de } \\
\text { manera subjetiva) }\end{array}$ & RDTO & Dependiente & Continua (acotada entre 1 y 7 ) \\
\hline
\end{tabular}

\section{ESTUDIO EMPÍRICO}

\subsection{La muestra: descripción y representatividad}

El trabajo de campo fue realizado entre los meses de abril y septiembre de 2006, y a la conclusión del mismo habíamos recibido un total de 56 cuestionarios válidos, lo que supone una tasa de respuesta del $21 \%$ sobre el total de la población objetivo. En el Cuadro 2 se puede observar la distribución de la población objetivo y de la muestra por sectores de actividad, sus correspondientes subsectores y para éstos últimos, además se indica entre paréntesis, el número de establecimientos franquiciados que los integran. En el Cuadro 3 recogemos qué subsectores requieren desempeñar actividades de transformación física y en cuáles se llevan a cabo solamente actividades comerciales. 
Cuadro 2: Distribución sectorial de la población objetivo y de la muestra

\begin{tabular}{|c|c|c|c|c|c|}
\hline \multirow{2}{*}{ SECTORES } & \multicolumn{2}{|c|}{ POBLACIÓN } & \multicolumn{3}{|r|}{ MUESTRA } \\
\hline & $\mathbf{N}$ & $\%$ & $\mathbf{N}$ & $\%$ & SUBSECTORES (n) \\
\hline Servicios & 129 & $48^{\prime} 31$ & 25 & $44^{\prime} 65$ & $\begin{array}{c}\text { Agencias inmobiliarias (3) } \\
\text { Centros de estética y belleza (2) } \\
\text { Clínicas odontológicas (1) } \\
\text { Compra - Venta artículos usados (1) } \\
\text { Enseñanza (1) } \\
\text { Servicio financiero (2) } \\
\text { Tintorería y lavandería (15) }\end{array}$ \\
\hline $\begin{array}{l}\text { Comercio } \\
\text { Minorista }\end{array}$ & 56 & $20^{\prime} 98$ & 11 & $19^{\prime} 64$ & $\begin{array}{c}\text { Dietética, herbolarios y parafarmacia (1) } \\
\text { Fotografía (1) } \\
\text { Juguetería (1) } \\
\text { Mobiliario (2) } \\
\text { Moda (1) } \\
\text { Oficina y papelería (1) } \\
\text { Panadería y pastelería (1) } \\
\text { Tienda de vinos (1) } \\
\text { Tienda especializada (2) }\end{array}$ \\
\hline $\begin{array}{c}\text { Hostelería y } \\
\text { Restauración }\end{array}$ & 82 & $30^{\prime} 71$ & 20 & $35^{\prime} 71$ & $\begin{array}{l}\text { Cafeterías (1) } \\
\text { Comida rápida (15) } \\
\text { Heladerías (1) } \\
\text { Italianos (1) } \\
\text { Tapas (1) } \\
\text { Temáticos (1) }\end{array}$ \\
\hline TOTAL & 267 & 100 & 56 & 100 & \\
\hline
\end{tabular}

Cuadro 3: Distribución de las respuestas según el tipo de actividad

\begin{tabular}{||c|c||}
\hline ACTIVIDAD & SUBSECTORES \\
\hline \begin{tabular}{||c|}
\hline Transformación y \\
Comercialización
\end{tabular} & $\begin{array}{c}\text { centros de estética y belleza, clínicas odontológicas, tintorería y lavandería, } \\
\text { fotografía, panadería y pastelería, cafeterías, comida rápida, heladerías, } \\
\text { italianos, tapas y temáticos }\end{array}$ \\
\hline Comercialización & $\begin{array}{c}\text { agencias inmobiliarias, compra-venta artículos usados, enseñanza, servicio } \\
\text { financiero, dietética, herbolarios y parafarmacia, juguetería, mobiliario, } \\
\text { moda, oficina y papelería, tienda de vinos, tienda especializada }\end{array}$ \\
\hline \hline
\end{tabular}

En cuanto al análisis de la representatividad de la muestra, calculamos y comparamos los porcentajes de respuestas recibidas por cada sector de actividad con los porcentajes de la población objetivo (Cuadro 2). Aunque éstos no son idénticos, sí son similares y mantienen la proporción, esto es, en la población objetivo y en la muestra, el sector servicios es el que tiene un mayor peso seguido de hostelería y restauración y comercio 
minorista. Por tanto, podemos aceptar que la muestra tiene un grado de representación sólido con respecto a la población objetivo, lo cual nos va a permitir utilizarla para realizar los análisis estadísticos pertinentes y poder generalizar las conclusiones al ámbito de estudio determinado.

4.2. Análisis de las escalas de medida: unidimensionalidad, fiabilidad y validez

Respecto a la unidimensionalidad, la única escala que plantea algún problema es la del rendimiento medido de forma subjetiva (RDTO). Para esta escala fue necesario realizar dos análisis factoriales puesto que en el primero dicha escala cargaba sobre dos factores. Una vez desglosada en dos la escala de medida, se confirmó el cumplimiento de la dimensionalidad. De este modo, a partir de este momento, los cálculos se realizarán teniendo en cuenta que la escala de medida del rendimiento 1 (RDTO 1) comprende la suma de las ponderaciones relativas a la importancia y satisfacción de los franquiciados en cuanto a las ventas, el tiempo de recuperación de la inversión y la productividad, mientras que la del rendimiento 2 (RDTO 2) incluye el sumatorio del producto de la importancia y satisfacción de los franquiciados respecto a la evolución del concepto de negocio, las condiciones de compra y aprovisionamiento y la equidad en el reparto de las ganancias.

Cuadro 4: Estudio de dimensionalidad y fiabilidad

\begin{tabular}{||c|c|c|c|c|c|c||}
\hline ESCALA & \multicolumn{3}{|c|}{ A. FACTORIAL 1 } & A. FACTORIAL 2 \\
\hline Variable & NTAC & VALOR & VTAS/m2 & RDTO & RDTO 1 & RDTO 2 \\
\hline $\mathbf{n}^{\circ}$ ítem & 5 & 2 & 1 & 6 & 3 & 3 \\
\hline KMO & $0^{\prime} 814$ & $0^{\prime} 500$ & - & $0^{\prime} 762$ & $0^{\prime} 629$ & $0^{\prime} 679$ \\
\hline signif. de Bartlett & $0^{\prime} 000$ & $0^{\prime} 000$ & - & $0^{\prime} 000$ & $0^{\prime} 000$ & $0^{\prime} 000$ \\
\hline $\mathbf{N}^{\circ}$ factores & 1 & 1 & - & 2 & 1 & 1 \\
\hline \% varianza explicada & $72^{\prime} 896$ & $88^{\prime} 380$ & - & $78^{\prime} 396$ & $71^{\prime} 563$ & $77^{\prime} 643$ \\
\hline Fiabilidad & $0^{\prime} 9055$ & $0^{\prime} 768^{* *}$ & - & - & $0^{\prime} 7921$ & $0^{\prime} 8555$ \\
\hline
\end{tabular}

** La correlación es significativa al nivel 0'01 bilateral

En el Cuadro 4 aparecen los resultados del análisis de la unidimensionalidad (método de componentes principales y varimax) así 
como la fiabilidad de las escalas de medida (correlaciones y $\alpha$ de Cronbach).

Para concluir con el análisis de la adecuación de las escalas de medida, estudiamos la validez de contenido, la validez convergente y la validez nomológica, llegando a la conclusión de que las escalas de medida de todas las variables son válidas.

\subsection{Descripción de las variables}

Mediante el cálculo del valor mínimo, máximo, la media y la desviación típica, obtenemos una descripción de las variables. Como podemos observar en el Cuadro 5 , en la que aparecen los estadísticos descriptivos antes mencionados, disponemos de 56 observaciones para la mayoría de las variables. La excepción es para la variable VTAS/m2, que por tratarse de datos relativos al rendimiento, algunos franquiciados, en concreto diez, prefirieron no aportarlos. Finalmente, el Cuadro 6 recoge las correlaciones entre las variables.

Cuadro 5: Estadísticos descriptivos

\begin{tabular}{||c|c|c|c|c|c||}
\hline Variable & $\mathbf{N}$ & MIN & MÁX & MEDIA & DESV. TÍPICA \\
\hline NTAC & 56 & 5 & 30 & $16^{\prime} 79$ & $6^{\prime} 97$ \\
\hline VALOR & 56 & 2 & 14 & $8 ' 25$ & $3{ }^{\prime} 71$ \\
\hline VTAS/m2 & 46 & $197^{\prime} 33$ & $10542^{\prime} 86$ & $3182^{\prime} 7588$ & $2362^{\prime} 5308$ \\
\hline RDTO 1 & 56 & 3 & 147 & $69^{\prime} 14$ & $33^{\prime} 81$ \\
\hline RDTO 2 & 56 & 3 & 147 & $48^{\prime} 27$ & $30^{\prime} 08$ \\
\hline
\end{tabular}

Cuadro 6: Correlaciones

\begin{tabular}{||c|c|c|c|c||}
\hline \hline Variable & VALOR & VTAS/m2 & RDTO 1 & RDTO 2 \\
\hline NTAC & $0^{\prime} 709^{* * *}$ & $0^{\prime} 239$ & $0^{\prime} 150$ & $0^{\prime} 261^{*}$ \\
\hline VALOR & & $0^{\prime} 339^{* *}$ & $0^{\prime} 130$ & $0^{\prime} 473^{* * *}$ \\
\hline
\end{tabular}

*** la correlación es significativa al nivel 0'01 (bilateral)

** la correlación es significativa al nivel 0'05 (bilateral)

* la correlación es significativa al nivel 0'10 (bilateral) 


\subsection{Contraste del modelo teórico}

En primer lugar, hemos analizado el posible sesgo de no respuesta comparando los aspectos señalados por los establecimientos que han respondido el cuestionario en las primeras semanas con aquéllos que contestaron en las últimas semanas, sin encontrar diferencias significativas.

Para poder contrastar la primera hipótesis, y tal y como están definidas las variables implicadas en el estudio, se han formulado los siguientes tres modelos:

$$
\begin{aligned}
& \text { Modelo 1.1: VTAS } / \mathrm{m} 2=B_{0}+B_{1} \text { NTAC }+B_{2} \text { TRANSF }+\varepsilon_{\mathrm{t}} \\
& \text { Modelo 1.2: } \\
& \text { RDTO1 }=B_{0}+B_{1} \text { NTAC }+B_{2} \text { TRANSF }+\varepsilon_{t} \\
& \text { Modelo 1.3: } \text { RDTO2 }=B_{0}+B_{1} \text { NTAC }+B_{2} \text { TRANSF }+\varepsilon_{t}
\end{aligned}
$$

Para la segunda hipótesis planteada, los tres modelos a contrastar serían los siguientes:

Modelo 2.1: VTAS $/ m 2=B_{0}+B_{1}$ VALOR $+B_{2}$ TRANSF $+\varepsilon_{t}$

Modelo 2.2: RDTO1 $=B_{0}+B_{1}$ VALOR $+B_{2}$ TRANSF $+\varepsilon_{t}$

Modelo 2.3: RDTO2 $=B_{0}+B_{1}$ VALOR $+B_{2}$ TRANSF $+\varepsilon_{t}$

Finalmente, para poder contrastar la tercera hipótesis, se han formulado los modelos que se exponen a continuación:

$$
\begin{aligned}
& \text { Modelo 3.1: VTAS } / \mathrm{m} 2=B_{0}+B_{1} \text { NTAC }+B_{2} \text { VALOR }+B_{3} \text { TRANSF }+\varepsilon_{t} \\
& \text { Modelo 3.2: } \text { RDTO1 }=B_{0}+B_{1} \text { NTAC }+B_{2} \text { VALOR }+B_{3} \text { TRANSF }+\varepsilon_{t} \\
& \text { Modelo 3.3: } \text { RDTO2 }=B_{0}+B_{1} \text { NTAC }+B_{2} \text { VALOR }+B_{3} \text { TRANSF }+\varepsilon_{t}
\end{aligned}
$$

A continuación, para contrastar los modelos planteados se recurrió al análisis de regresión lineal, empleando en todos los casos, el programa estadístico SPSS para Windows versión 15.0. Los resultados de las regresiones lineales realizadas para los modelos $1.1,1.2$ y 1.3 (modelos cuya variable independiente es la naturaleza tácita) aparecen en el Cuadro 7. 
Cuadro 7: Resumen de los modelos de regresión para la naturaleza tácita

\begin{tabular}{|c|c|c|c|c|}
\hline & & $\begin{array}{c}\text { Mod 1.1 } \\
\text { VTAS/m2 }\end{array}$ & $\begin{array}{l}\text { Mod } 1.2 \\
\text { RDTO } 1\end{array}$ & $\begin{array}{l}\text { Mod } 1.3 \\
\text { RDTO } 2\end{array}$ \\
\hline \multirow[t]{2}{*}{ (Constante) } & $\boldsymbol{\beta}_{0}$ & $2654^{\prime} 932^{* *}$ & $55^{\prime} 879 * * *$ & $25^{\prime} 079^{* *}$ \\
\hline & Error típico & $1069^{\prime} 640$ & $12^{\prime} 438$ & $10^{\prime} 608$ \\
\hline \multirow[t]{2}{*}{ NTAC } & $\beta_{1}$ & $100 ' 532 *$ & $0^{\prime} 650$ & $0^{\prime} 814$ \\
\hline & Error típico & $50^{\prime} 902$ & $0^{\prime} 705$ & $0^{\prime} 601$ \\
\hline \multirow[t]{2}{*}{ TRANSF } & $\boldsymbol{\beta}_{2}$ & $-1598^{\prime} 692^{*}$ & $3 ' 290$ & $13 ' 330$ \\
\hline & Error típico & $814^{\prime} 079$ & $10^{\prime} 778$ & 9'192 \\
\hline \multicolumn{2}{|c|}{$\mathbf{R}^{2}$ ajustado } & 0'094 & $-0^{\prime} 013$ & $0^{\prime} 070$ \\
\hline \multicolumn{2}{|c|}{ Error típico de la estimación } & $2248^{\prime} 2639$ & $34^{\prime} 02$ & $29^{\prime} 01$ \\
\hline \multicolumn{2}{|c|}{ Test F } & $3^{\prime} 345^{* *}$ & $0^{\prime} 657$ & $3^{\prime} 065^{*}$ \\
\hline
\end{tabular}

$* * * \mathrm{p} \leq 0^{\prime} 01 ;{ }^{* *} \mathrm{p} \leq 0^{\prime} 05 ; * \mathrm{p} \leq 0^{\prime} 1$

Si observamos la prueba $F$, sólo los modelos estimados para las ventas por metro cuadrado (modelo 1.1) y el rendimiento 2 (modelo 1.3) son significativos, siendo los resultados obtenidos fiables puesto que ambos modelos cumplen los supuestos estadísticos básicos de normalidad, homocedasticidad, linealidad, no autocorrelación y ausencia de multicolinealidad. La variable NTAC explica un $9,4 \%$ y un $7 \%$ de la variación de las ventas por metro cuadrado y del rendimiento 2 respectivamente.

Cuadro 8: Resumen de los modelos de regresión para el valor

\begin{tabular}{|c|c|c|c|c|}
\hline & & $\begin{array}{c}\text { Mod } 2.1 \\
\text { VTAS/m2 }\end{array}$ & $\begin{array}{l}\text { Mod } 2.2 \\
\text { RDTO } 1\end{array}$ & $\begin{array}{l}\text { Mod } 2.3 \\
\text { RDTO } 2\end{array}$ \\
\hline \multirow[t]{2}{*}{ (Constante) } & $\boldsymbol{\beta}_{0}$ & $2184^{\prime} 316^{* *}$ & $58^{\prime} 249^{* * *}$ & $14^{\prime} 654$ \\
\hline & Error típico & $996 ' 381$ & $11^{\prime} 711$ & $9^{\prime} 225$ \\
\hline \multirow[t]{2}{*}{ VALOR } & $\beta_{1}$ & $266^{\prime} 100^{* * *}$ & $1^{\prime} 011$ & $3^{\prime} 521 * * *$ \\
\hline & Error típico & $94 ' 328$ & 1'352 & $1^{\prime} 065$ \\
\hline \multirow[t]{2}{*}{ TRANSF } & $\boldsymbol{\beta}_{2}$ & $-1710^{\prime} 440^{* *}$ & $3^{\prime} 578$ & 6'387 \\
\hline & Error típico & $781^{\prime} 971$ & $10^{\prime} 999$ & $8^{\prime} 663$ \\
\hline \multicolumn{2}{|c|}{$R^{2}$ ajustado } & $0^{\prime} 166$ & $-0^{\prime} 018$ & 0'202 \\
\hline \multicolumn{2}{|c|}{ Error típico de la estimación } & $2156^{\prime} 9023$ & $344^{\prime} 11$ & $26 ' 87$ \\
\hline \multicolumn{2}{|c|}{ Test F } & $5 ' 495 * * *$ & $0 ' 510$ & $7{ }^{\prime} 972 * * *$ \\
\hline
\end{tabular}

*** $\mathrm{p} \leq 0^{\prime} 01 ;{ }^{* *} \mathrm{p} \leq 0^{\prime} 05 ; *^{*} \mathrm{p} \leq 0^{\prime} 1$ 
En cuanto a los coeficientes, la única variable representativa de la naturaleza tácita que resulta significativa es la relativa al modelo de regresión estimado para las ventas por metro cuadrado (modelo 1.1). Tal y como ha sido construida dicha variable, el resultado de la regresión indica que cuanto menos tácito sea el conocimiento, mayores son las ventas por metro cuadrado.

Los resultados de las regresiones lineales calculadas para los modelos $2.1,2.2$ y 2.3 (modelos cuya variable independiente es el valor del conocimiento) aparecen en el Cuadro 8.

La prueba $F$ nos indica que los modelos de regresión estimados para las ventas/m2 y el rendimiento 2 (modelos 2.1 y 2.3 ) son significativos y los resultados obtenidos son fiables puesto que ambos modelos cumplen los supuestos estadísticos básicos de normalidad, homocedasticidad, linealidad, no autocorrelación y ausencia de multicolinealidad. Pero en este caso, la variable VALOR es capaz de explicar un mayor porcentaje de la variación de las ventas por metro cuadrado, y del rendimiento 2 , de lo que lo hacía la naturaleza tácita. Así, a mayor valor del conocimiento, mayores ventas por metro cuadrado y mayor satisfacción del franquiciado respecto a la evolución del concepto de negocio, las condiciones de compra y aprovisionamiento y la equidad en el reparto de las ganancias.

Cuadro 9: Resumen de los modelos de regresión múltiple

\begin{tabular}{|c|c|c|c|c|}
\hline & & $\begin{array}{c}\text { Mod 3.1 } \\
\text { VTAS/m2 }\end{array}$ & $\begin{array}{l}\text { Mod } 3.2 \\
\text { RDTO } 1\end{array}$ & $\begin{array}{l}\text { Mod } 3.3 \\
\text { RDTO } 2\end{array}$ \\
\hline \multirow[t]{2}{*}{ (Constante) } & $\boldsymbol{\beta}_{0}$ & $2038^{\prime} 723^{*}$ & $55^{\prime} 382 * * *$ & $18^{\prime} 503^{*}$ \\
\hline & Error típico & $1084 ' 126$ & $12^{\prime} 839$ & $10^{\prime} 054$ \\
\hline \multirow[t]{2}{*}{ NTAC } & $\beta_{1}$ & $22^{\prime} 952$ & $0^{\prime} 534$ & $-0^{\prime} 718$ \\
\hline & Error típico & $63^{\prime} 467$ & $0^{\prime} 949$ & $0^{\prime} 743$ \\
\hline \multirow[t]{2}{*}{ VALOR } & $\boldsymbol{\beta}_{2}$ & $238 ' 209^{*}$ & 0'335 & $4^{\prime} 429 * * *$ \\
\hline & Error típico & $122 ' 594$ & 1'814 & $1^{\prime} 421$ \\
\hline \multirow[t]{2}{*}{ TRANSF } & $\beta_{3}$ & $-1730^{\prime} 387^{* *}$ & $2^{\prime} 840$ & 7'377 \\
\hline & Error típico & $791 ' 919$ & $11^{\prime} 147$ & $8^{\prime} 729$ \\
\hline \multicolumn{2}{|c|}{$\mathbf{R}^{2}$ ajustado } & $0^{\prime} 149$ & $-0^{\prime} 031$ & 0'201 \\
\hline \multicolumn{2}{|c|}{ Error típico de la estimación } & $2179^{\prime} 0385$ & $34 ' 33$ & $26^{\prime} 89$ \\
\hline \multicolumn{2}{|c|}{ Test $\mathbf{F}$} & $3 ' 633^{* *}$ & $0^{\prime} 441$ & $5^{\prime} 619 * * *$ \\
\hline
\end{tabular}

$* * * \mathrm{p} \leq 0^{\prime} 01 ; * * \mathrm{p} \leq 0^{\prime} 05 ; * \mathrm{p} \leq 0^{\prime} 1$ 
Finalmente, los resultados de las regresiones lineales estimadas conjuntamente para la naturaleza tácita del conocimiento y el valor (modelos 3.1, 3.2 y 3.3) aparecen en el Cuadro 9.

Nuevamente la prueba $F$ nos muestra que los únicos modelos significativos son aquéllos que tienen como variable dependiente las ventas por metro cuadrado ó el rendimiento 2 (modelos 3.1 y 3.3). Ambos modelos cumplen con los supuestos estadísticos básicos de normalidad, homocedasticidad, linealidad y no autocorrelación. Sin embargo, los resultados obtenidos en estos modelos deben interpretarse con cautela dado que no se verifica el supuesto de ausencia de multicolinealidad.

En efecto, la alta correlación entre las dos variables independientes (NTAC y VALOR) indica la existencia de una dependencia lineal entre las variables, pero este hecho se observa más claramente cuando calculamos la regresión lineal simple del valor del conocimiento (VALOR) en función de su naturaleza tácita (NTAC): la variable NTAC explica el $499^{\prime} \%$ de la varianza del VALOR siendo su coeficiente de regresión positivo y significativo al nivel del $1 \%\left(\beta=00^{\prime} 377\right.$; error típico $=$ 0'051), es decir, cuanto más explícito es el conocimiento, mayor es su valor. Por tanto, con esta metodología no es posible verificar el efecto conjunto de la naturaleza tácita y el valor del conocimiento sobre el rendimiento de los sistemas de franquicia.

Un resultado que se ha repetido en los tres análisis efectuados (Cuadros 7, 8 y 9) es el relacionado con la variable de control. Efectivamente, los resultados de las regresiones en aquellos modelos en los que el rendimiento del sistema de franquicia está medido mediante las ventas por metro cuadrado, muestran que el coeficiente de la variable transformación (TRANSF) es negativo y significativo, lo que indica, no sólo la existencia de diferencias respecto a las ventas por metro cuadrado entre los establecimientos que realizan actividades comerciales y aquéllos en los que se llevan a cabo tanto actividades comerciales como productivas, sino también que, en este último tipo de establecimientos, las ventas por metro cuadrado son menores.

En definitiva, las hipótesis 1 y 2 se contrastan parcialmente y no podemos afirmar que se verifica la hipótesis 3 , debido a que las dos variables independientes están correlacionadas. 


\section{CONCLUSIÓN}

Este trabajo se ha centrado en el proceso de transferencia de conocimiento. Concretamente se ha pretendido evaluar el efecto de dos características del conocimiento, que determinan la dificultad con la que se pretende transferir, en el rendimiento de los sistemas de franquicia.

Para alcanzar dicho propósito se elaboró un cuestionario que fue enviado a 267 establecimientos franquiciados localizados en España y que pertenecían a diferentes cadenas y sectores de actividad. Con los 56 cuestionarios recibidos se llevó a cabo un análisis de regresión lineal (tanto simple como múltiple) que nos permitiera contrastar las hipótesis planteadas.

Los resultados de las regresiones parecen indicar que la naturaleza tácita tiene una influencia negativa en el rendimiento, medido a través de las ventas por metro cuadrado. Esto significa que cuanto más explícito (o menos tácito) sea el conocimiento que el franquiciador transmite a sus franquiciados, más facilidad tienen estos últimos para asimilarlo y poder reproducir de forma exacta el concepto de negocio. Así, el franquiciado podrá suministrar la oferta de productos a la que los clientes esperan acceder, y éstos verán cumplidas sus expectativas, por lo que se traducirá en unas mayores ventas por metro cuadrado.

En cuanto al valor del conocimiento, los resultados establecen una relación positiva con las ventas por metro cuadrado y con el rendimiento 2 . Cuanto más valioso y útil es el conocimiento, los franquiciados son más conscientes de la relevancia del mismo para poder desarrollar la actividad principal objeto de la franquicia y así replicar el éxito alcanzado por el franquiciador. La asimilación de ese conocimiento dará lugar a productos que llevan incorporados el saber hacer del franquiciador, de tal manera que va a ser difícil encontrar en el mercado otra organización que distribuya el mismo producto. Esto se traduciría en unas mayores ventas por metro cuadrado, mayor satisfacción de los franquiciados en relación a la evolución del concepto de negocio, las condiciones de compra y aprovisionamiento y la equidad en el reparto de las ganancias.

A pesar de estos resultados, no disponemos de evidencia empírica que nos permita establecer la influencia individual de la naturaleza tácita y el valor del conocimiento sobre el rendimiento 1 , el cual comprendía la satisfacción del franquiciado respecto a las ventas, el tiempo de recuperación de la inversión y la productividad. Y tampoco, 
podemos demostrar que el efecto combinado de ambas variables influye en el rendimiento de los sistemas de franquicia, aunque en este caso por diferente motivo: la correlación entre las variables independientes.

Finalmente, los resultados muestran diferencias en el rendimiento en función de si se realizan en el establecimiento sólo actividades comerciales o, además, se llevan a cabo actividades productivas. En concreto, las ventas por metro cuadrado son menores en aquellos establecimientos en donde se efectúan actividades productivas y comerciales lo cual creemos que puede ser debido a que 1) parte de la superficie del local se dedica a actividades de transformación y, por tanto, las dimensiones del establecimiento son necesariamente mayores, y 2) a que las desviaciones respecto a los procedimientos operativos establecidos por el franquiciador pueden ser mayores y ello repercute en las ventas por metro cuadrado.

Estos resultados deben ser valorados con precaución y prudencia dado que esta investigación presenta una serie de limitaciones. Así, en primer lugar, decir que el método utilizado para la recogida de información ha sido la encuesta, pero es que las características de las variables analizadas nos impedía la utilización de fuentes secundarias. En segundo lugar, estos resultados sólo son generalizables a nuestra población de referencia. En tercer lugar, nuestra muestra es pequeña, 56 establecimientos franquiciados, pero hay que tener en cuenta que nuestra población o universo son los 267 franquiciados asociados a la AEDEF y que por tanto la tasa de respuesta es de un $21 \%$, una tasa alta teniendo en cuenta los inconvenientes de utilizar el cuestionario como método de recogida de información. Finalmente, hemos utilizado una única fuente de información ya que el cuestionario sólo fue enviado a los franquiciados que, en realidad, son una parte de la relación diádica que se produce en la franquicia. Sin embargo, considerábamos relevante que la información nos la proporcionara un agente externo que estuviera directamente relacionado con dicho conocimiento.

A pesar de estas limitaciones, entendemos que los resultados obtenidos en esta investigación son satisfactorios por varios motivos. Por un lado, demostramos la influencia que la naturaleza tácita y el valor del conocimiento tienen sobre el rendimiento cuando la literatura se ha centrado en el estudio de la relación indirecta a través del proceso de transferencia. Por otro lado, el estudio se aplica al caso concreto de los sistemas de franquicia y si bien la literatura en esta materia se ha centrado en el estudio de las decisiones de franquiciar o internalizar y la 
perspectiva de análisis más utilizada es la del franquiciador, en este trabajo hemos tratado aspectos relacionados con la competitividad de los sistemas de franquicia desde la punto de vista del franquiciado.

Otra aportación de este trabajo es la construcción de los indicadores empleados para medir el rendimiento de los sistemas. No sólo hemos considerado una medida objetiva, las ventas por metro cuadrado, sino que también utilizamos una medida basada en valoraciones dadas por los franquiciados a unos aspectos del rendimiento de su establecimiento.

Las conclusiones de este trabajo pueden ser de utilidad tanto a franquiciados como a franquiciadores. De una parte, puede servir a los primeros para exigir al franquiciador una mayor descripción del conocimiento y un mayor esfuerzo en desarrollar un conocimiento que les permita explotar su establecimiento de tal forma que sus resultados sean, al menos, los mismos que los de otros establecimientos del sistema, pero superiores a los de la competencia. De otra parte, puede servirle al franquiciador a la hora de planificar los recursos necesarios para describir y desarrollar el conocimiento que necesitan sus franquiciados.

\section{BIBLIOGRAFÍA}

Alonso Prieto, M. (2003) La Franquicia de la 'A' a la 'Z'. Manual para el Franquiciador y el Franquiciado. Madrid: LID Editorial Empresarial.

AmBrosinI, V. and C. Bowman (2001) "Tacit Knowledge: Some Suggestions for Operationalization", Journal of Management Studies, 38, no. 6, pp. 811-829.

ANDERSON, E.E. (1984) "The Growth and Performance of Franchise Systems: Company versus Franchisee Ownership", Journal of Economics and Business, 36, pp. 421-431.

BATES, A. (2002) "What Drives Profit in Franchising", Franchising World, 34 , no. 3, pp. 46-47.

BRADACH, J.L. (1998) Franchise Organizations. Massachusetts: Harvard Business School Press.

BRESMAN, H.; J. BIRKINSHAW and R. NoBEL (1999) "Knowledge Transfer in International Acquisitions", Journal of International Business Studies, 30, no. 3, pp. 439-462. 
CARMAN, J.M. and T.A. KLEIN (1986) "Power, Property and Performance in Franchising: A Review and Integration of Theory", Research in Marketing, 8, pp. 71-130.

CAStRogiovanNI, G.J.; R.T. JUSTIS and S.D. JULIAN (1993) "Franchise Failure Rates: An Assessment of Magnitude and Influencing Factors", Journal of Small Business Management, April, pp. 105-114.

CAVES, R.E. and W.F. MURPHY II (1976) "Franchising: Firms, Markets and Intangible Assets", Southern Economic Journal, 42, pp. 572-586.

CHEN, C.J. (2004) "The Effects of Knowledge Attribute, Alliance Characteristics and Absorptive Capacity on Knowledge Transfer Performance", R\&D Management, 34, no. 3, pp. 311-321.

CONTRACTOR, F.K. and S.K. KUNDU (1998) "Modal Choice in a World of Alliances: Analyzing Organizational Forms in the International Hotel Sector", Journal of International Business Studies, 29, no. 2, pp. 325358.

COWAN, R.; L. SOETE and O. TCHERVONNAYA (2001) Knowledge Transfer and the Services Sector in the Context of the New Economy. Maastricht: MERIT - Maastricht Economic Research Institute on Innovation and Technology.

DARR, E.D.; L. ARGOTE and D. EPPLE (1995) "The Acquisition, Transfer and Depreciation of Knowledge in Service Organizations: Productivity in Franchises", Management Science, 41, no. 11, pp. 1750-1762.

DavenPoRT, T.H. and L. Prusak (2001) Conocimiento en Acción. Cómo las Organizaciones Manejan lo que Saben. Buenos Aires: Pearson Educación.

Dixon, N.M. (2001) El Conocimiento Común. Cómo Prosperan las Compañías que Comparten lo que Saben. México: Oxford University Press.

DYER, J.H. and K. NOBEOKA (2000) "Creating and Managing a HighPerformance Knowledge Sharing Network: The Toyota Case", Strategic Management Journal, 21, pp. 345-367.

ElanGO, B. and V.H. FRIED (1997) "Franchising Research: A Literature Review and Synthesis", Journal of Small Business Management, July, pp. 68-81.

EMPSON, L. (2001) "Fear of Exploitation and Fear of Contamination: Impediments to Knowledge Transfer in Mergers between Professional Service Firms", Human Relations, 54, no. 7, pp. 839-862.

Fenwick, G.D. and M. STROMBOM (1998) "The Determinants of Franchisee Performance: An Empirical Investigation", International Small Business Journal, 16, no. 4, pp. 28-45. 
Flechoso SIeRRA, J.J. (1997) El Libro de la Franquicia. Madrid: Editorial Biblioteca Nueva.

Foss, N.J. and T. PeDERSEN (2002) "Transferring Knowledge in MNC's: The Roles of Sources of Subsidiary Knowledge and Organizational Context", Journal of International Management, 8, no. 1, pp. 49-67.

GALUNIC, D.C. and S. RODAN (1998) "Resource Recombinations in the Firm: Knowledge Structures and the Potential for Schumpeterian Innovation", Strategic Management Journal, 19, pp. 1193-1201.

GARUD, R. and P.R. NAYYAR (1994) "Transformative Capacity: Continual Structuring by Intertemporal Technology Transfer", Strategic Management Journal, 15, pp. 365-385.

GRANT, R.M. (1996a) "Prospering in Dynamically-Competitive Environments: Organizational Capability as Knowledge Integration", Organization Science, 7, no. 4, pp. 375-387.

- (1996b) "Toward a Knowledge-Based Theory of the Firm", Strategic Management Journal, 17, pp. 109-122.

- (1997) "The Knowledge-Based View of the Firm: Implications for Management Practice", Long Range Planning, 30, $\mathrm{n}^{\circ}$ 3, pp. 450-454.

GuPTA, A.K. and V. GovindARAJAN (2000) "Knowledge Flows within Multinational Corporations", Strategic Management Journal, 21, pp. 473-496.

HALL, R. and P. ANDRIANI (2002) "Managing Knowledge for Innovation", Long Range Planning, 35, pp. 29-48.

HART, S. and C. BAnBury (1994) "How Strategy-Making Processes Can Make a Difference", Strategic Management Journal, 15, no. 4, pp. 251-269.

HedLuND, G. (1994) "A Model of Knowledge Management and the N-Form Corporation", Strategic Management Journal, 15, pp. 73-90.

HIPPEL, E. von (1994) '"Sticky Information' and the Locus of Problem Solving: Implications for Innovation", Management Science, 40, no. 4, pp. 429-439.

HOLTSHOUSE, D. (1998) "Knowledge Research Issues", California Management Review, 40, no. 3, pp. 277-280.

Husted, K. and S. MiCHAILOVA (2002) "Diagnosing and Fighting KnowledgeSharing Hostility", Organizational Dynamics, 31, no. 1, pp. 60-73.

KotABE, M.; X. MARTIN and H. DOMOTO (2003) "Gaining from Vertical Partnerships: Knowledge Transfer, Relationship Duration and Supplier 
Performance Improvement in the U.S. and Japanese Automotive Industries", Strategic Management Journal, 24, pp. 293-316.

KROGH, G. von; I. NONAKA and M. ABEN (2001) "Making the Most of Your Company's Knowledge: A Strategic Framework", Long Range Planning, 34, pp. 421-439.

LUBIT, R. (2001) "Tacit Knowledge and Knowledge Management: The Keys to Sustainable Competitive Advantage", Organizational Dynamics, 29, no. 4, pp. 164-178.

MALeRBA, F. and L. OrSENIGO (2000) "Knowledge, Innovative Activities and Industrial Evolution", Industrial and Corporate Change, 9, no. 2, pp. 289-314.

MARTIN, R.E. (1988) "Franchising and Risk Management", The American Economic Review, 78, no. 5, pp. 954-968.

- and R.T. JUSTIS (1993) "Franchising, Liquidity Constraints and Entry", Applied Economics, 25, pp. 1269-1277.

NIELSEN, B.B. (2001) "The Role of Knowledge Embeddedness in the Process of Creation of Synergies in International Strategic Alliances", Working Paper, no. 7. Copenhagen Business School.

NonaKA, I. (1991) "The Knowledge-Creating Company", Harvard Business Review, 69, no. 6, pp. 96-104.

-, P. Reinmoller and D. SEnoo (1998) "The 'ART' of Knowledge: Systems to Capitalize on Market Knowledge", European Management Journal, 16, no. 6, pp. 673-684.

- and H. TAKEUCHI (1995) The Knowledge-Creating Company. New York: Oxford University Press.

-, R. TOYAMA and N. KonNo (2000) "SECl, Ba and Leadership: A Unified Model of Dynamic Knowledge Creation", Long Range Planning, 33, pp. 5-34.

NORTON, S.W. (1988a) "An Empirical Look at Franchising as an Organizational Form", Journal of Business, 61, no. 2, pp. 197-218.

- (1988b) "Franchising, Brand Name Capital and the Entrepreneurial Capacity Problem", Strategic Management Journal, 9, pp. 105-114.

O'DeLL, C. and C.J. GRAYSON (1998) "If Only we Knew What we Know: Identification and Transfer of Internal Best Practices", California Management Review, 40, no. 3, pp. 154-174. 
OSterloh, M. and B.S. FreY (1999) "Motivation, Knowledge Transfer and Organizational Form", Working Paper, no. 27. University of Zurich: Institute for Empirical Research in Economics.

PABloS, S. de (1998) Franquicias. Cómo montar un negocio de éxito. Madrid: Ediciones Temas de Hoy.

Polanyl, M. (1962) Personal Knowledge: Towards a Post-Critical Philosophy. Gloucester: University of Chicago Press.

- (1966) The Tacit Dimension. Gloucester: Peter Smith.

SCHULZ, M. (2001) "The Uncertain Relevance of Newness: Organizational Learning and Knowledge Flows", Academy of Management Journal, 44, no. 4, pp. 661-681.

- and L.A. JOBE (2001) "Codification and Tacitness as Knowledge Management Strategies. An Empirical Exploration", Journal of High Technology Management Research, 12, pp. 139-165.

SIMONIN, B.L. (1999) "Ambiguity and the Process of Knowledge Transfer in Strategic Alliances", Strategic Management Journal, 20, pp. 595-623.

SORENSON, O. and J.B. SøRENSEN (2001) "Finding the Right Mix: Franchising, Organizational Learning and Chain Performance", Strategic Management Journal, 22, pp. 713-724.

Subramaniam, M. and N. VenkATRAMAN (2001) "Determinants of Transnational New Product Development Capability: Testing the Influence of Transferring and Deploying Tacit Overseas Knowledge", Strategic Management Journal, 22, pp. 359-378.

SZULANSKI, G. (1996) "Exploring Internal Stickiness: Impediments to the Transfer of Best Practice within the Firm", Strategic Management Journal, 17, pp. 27-43.

-, R.J. JENSEN and T. LeE (2001) "On Re-using Knowledge: The Consequences of Adaptation of Franchising Knowledge for Local Network Growth", Working Paper, no. 10. University of Pensylvania: Reginald H. Jones Center.

TeECE, D. (2000) "Strategies for Managing Knowledge Assets: The Role of Firm Structure and Industrial Context", Long Range Planning, 33, pp. 35-54.

Thompson, R.S. (1994) "The Franchise Life Cycle and the Penrose Effect", Journal of Economic Behaviour and Organization, 24, no. 2, pp. 207218.

TORMO, E. A. (2002) De Emprendedor a Franquiciador. Madrid: Ediciones Selina Olmedo. 
TSAI, W. (2001) "Knowledge Transfer in Intraorganizational Networks: Effects of Network Position and Absorptive Capacity on Business Unit Innovation and Performance", Academy of Management Journal, 44, no. 5, pp. 996-1004.

WINTER, S.G. (1987) "Knowledge and Competence as Strategic Assets". T. TEECE (ed.) The Competitive Challenge: Strategies for Industrial Innovation and Renewal. Cambridge: Ballinger Publishing.

ZANDER, U. and B. KOGUT (1995) "Knowledge and the Speed of the Transfer and Imitation of Organizational Capabilities: An Empirical Test", Organization Science, 6, no. 1, pp. 76-92. 